\title{
Empty nose syndrome treated by autologous auricular cartilage turbinate augmentation: a case report
}

\author{
Tzu-I Wu, MD' ${ }^{1}$, Chung-Yu Hao, MD ${ }^{1}$ id, Yu-Hsun Chiu, MD, MMS ${ }^{1,2,3}$ \\ 'Department of Otolaryngology, Head and Neck Surgery, Shin Kong Wu-Ho-Su Memorial Hospital, Taipei, Taiwan \\ ${ }^{2}$ Department of Otolaryngology, Head and Neck Surgery, National Taiwan University Hospital, Taipei, Taiwan \\ ${ }^{3}$ Institute of Biomedical Engineering, National Taiwan University, Taipei, Taiwan
}

Empty nose syndrome (ENS), once viewed as a psychological issue, is now a recognized complication of turbinate surgery. It is most frequently associated with total turbinate excision. Its symptoms include chronic dryness of the nose and pharynx, paradoxical obstruction of the nose, feeling of suffocation, lack of airflow sensation, and even neuropathic pain. It may cause severe stress upon the patient resulting in mood instability issues. Surgical treatment to augment turbinate volume has been reported to be an effective treatment method. We would like to report a case of ENS treated by autologous auricular cartilage turbinate augmentation.

Keywords: ear cartilage; nasal cavity; natural orifice endoscopic surgery; turbinates; unilateral nasal obstruction

\section{Introduction}

The term empty nose syndrome (ENS) was first coined by Kern and Stenkvist in 1994. Although once considered a psychological issue, it is now a recognized complication of turbinate surgery, most frequently following total turbinate excision. The symptoms of ENS include chronic dryness of the nose and pharynx, paradoxical obstruction of the nose, feeling of suffocation, lack of airflow sensation, and neuropathic pain. Nasal humidification, patient education, and treatment of concomitant medical conditions (e.g., depression) form the first line of treatment. Surgical treatment to augment the turbinate volume has been reported to be an effective method. Herein, we report a case of ENS treated by autologous auricular cartilage turbinate augmentation.

\section{Case report}

A 63-year-old female presented to our ENT (ear, nose, throat) clinic with complaints of severe nasal obstruction and nasal dryness. She denied a history of any systemic illness. Her symptoms included nasal obstruction, dryness, and nasal pain, especially on the left side. She had nasal discomfort since approximately 8 years, when she first underwent inferior turbinate laser ablation surgery that provided partial relief for about a year. The patient another turbinate surgery and septoplasty in 2012, however, the symptoms relapsed shortly, and she underwent a third turbinate surgery after a few months.

Despite having undergone multiple surgeries involving the nasal turbinates in the past decade, the patient continued to suffer from a sensation of nasal blockage, left nasal pain that increased in severity on inspiration, and frequent dry crusting. Physical examination upon initial presentation at our clinic re- 

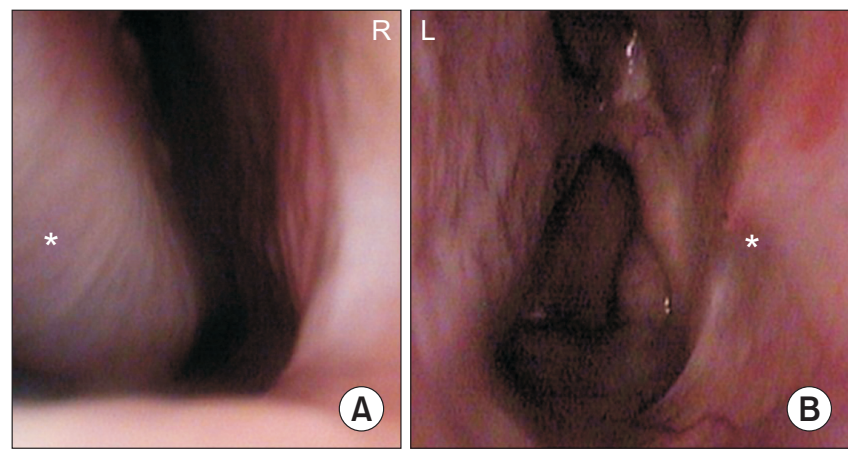

Fig. 1. Fiberscopic images of the right (A) and left (B) nasal cavities. Note the difference between the bilateral lateral walls, depicted as asterisks, due to a lack of inferior turbinate in the left nasal cavity.

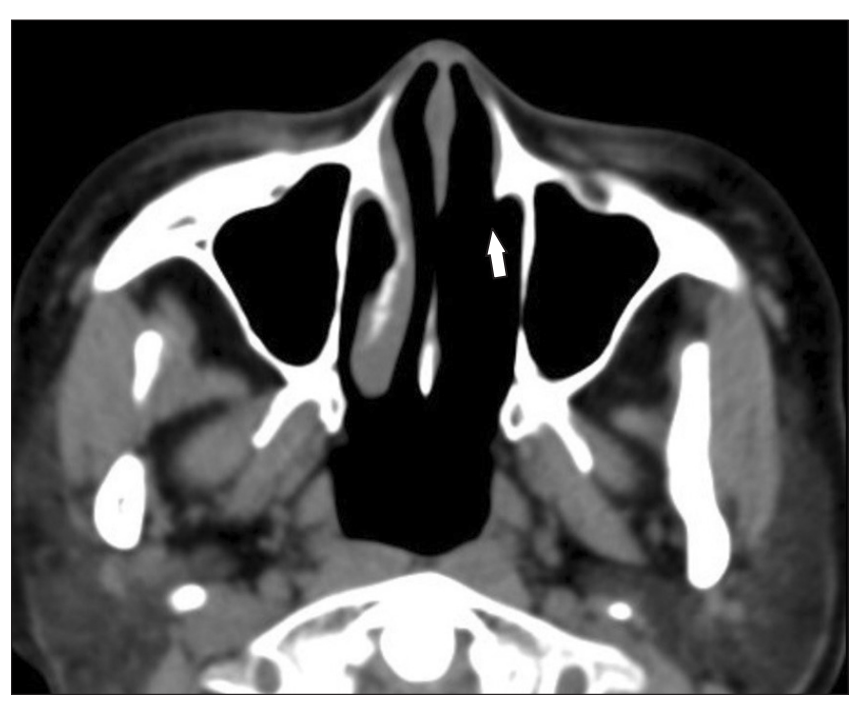

Fig. 2. Non-contrast enhanced computed tomography of paranasal sinuses revealed a lack of inferior nasal conchae in the patient's left nasal cavity (white arrow).

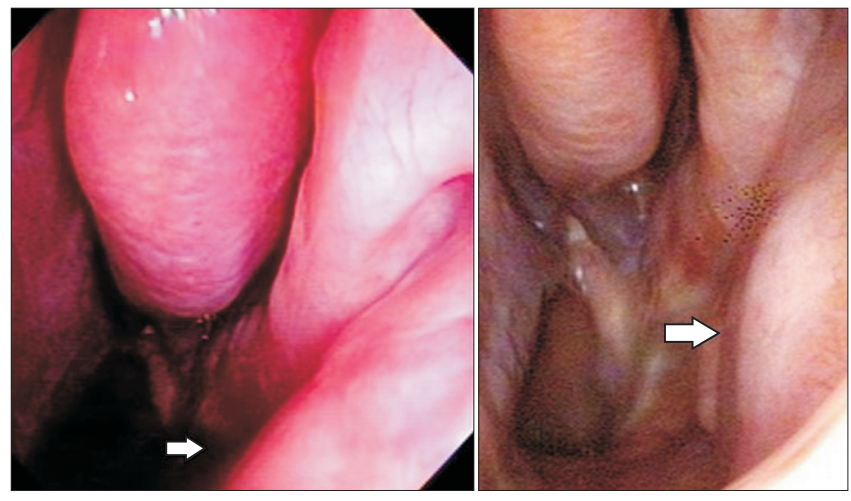

Fig. 3. Fiberscope examination showed good recovery of the mucosal wound and no signs of dislocation or infection of the implanted cartilage (white arrows). vealed extremely patent nasal cavities bilaterally with mild mucoid discharge and minimal crusting. Bosmine-soaked cotton pledge packing did not significantly relieve the symptoms. The patient was prescribed mometasone furoate nasal spray and mucolytics for 2 weeks. The patient denied any improvement in her nasal symptoms upon follow-up. Further fiberscope examination (Fig. 1) and non-contrast computed tomography of the paranasal sinuses revealed a lack of inferior nasal concha in her left nasal cavity (Fig. 2). Cotton test was performed by placing two dry cotton buds in the lateral-inferior quadrant of each nasal cavity for several minutes. The test was considered positive as it resulted in subjective improvement, but only on the left side. Considering a diagnosis of ENS on the left side, the patient underwent left inferior turbinate reconstruction with autologous conchal cartilage implantation in May 2018. Weekly postoperative follow-up revealed good recovery of the wound. Furthermore, the patient reported significant improvement in her nasal symptoms 18 days after the surgery. Fiberscope examination showed good recovery of the mucosal wound and no signs of dislocation or infection of the implanted cartilage (Fig. 3). One month after the surgery, the patient denied recurrence of her previous nasal distress, and remained symptom-free until the last follow-up in January 2019, 6 months after the treatment.

\section{Discussion}

ENS was first diagnosed by Eugene Kern and Monika Stenkvist in 1994 and is essentially a diagnosis of exclusion [1,2]. ENS is an iatrogenic disorder frequently recognized based on the presence of paradoxical nasal obstruction despite an objectively wide and patent nasal fossa [1].

ENS is a potentially devastating complication of nasal surgery, which often results in inability to concentrate, chronic fatigue, frustration, irritability, anger, anxiety, depression, and even suicide.

The nose provides more than $50 \%$ of the overall airflow resistance, and the turbinates are the recognition sites for airflow sensation [1-3]. Manometric studies or acoustic rhinometry generally report a fully patent airway in patients with ENS; however, the findings may be coupled with below-normal rates of resistance. Drastically low respiratory resistance might considerably upset the equilibrium required for deep pulmonary inspiration and may contribute to a subjective sense of inadequate nasal flow and subsequent nasal congestion $[1,4]$. The loss of turbinate tissue disrupts airflow within the nose, which may be perceived as poor nasal breathing [1]. 
The principles of management of ENS are based on replacement of missing tissue, rehabilitation of airway resistance, and control of symptoms [4]. Both medical and surgical modalities are available for the treatment of ENS. Conservative treatments include nasal saline irrigation, use of nasal moisturizing ointments, and plugging the nasal cavity; however, are usually less effective.

The surgical treatment of ENS was first described by Houser [1] in 2007. The group treated patients with submucosal implantation of acellular dermis, and all patients reported subjective improvement, as demonstrated by a significant decrease in the mean SNOT-25 score (58 to 38).

The goals of surgical treatment for ENS are to rebuild the internal nasal structures and narrow the airway in order to provide nasal resistance, promote the regeneration of normal mucosa, facilitate moisture retention in the nasal cavity by reducing airflow, and improve the vascular supply of the nasal cavity [4].

Performing the cotton test prior to implantation is crucial, as it can temporarily alter the nasal airflow and permit assessment of the patient's subjective response to decide the feasible location of the implant. The cotton test was validated in a study using the empty nose syndrome 6 item questionnaire (ENS6Q) [5].

There has not been a standardized testing procedure as of the timing of this writing. We have proposed a method to perform the cotton test to minimize the differences between physicians and practitioners:

1. The patient is first asked to breathe comfortably for several minutes without any cotton pledges placed inside the nostril to provide information on baseline nasal symptoms.

2. The patient is then asked to breathe comfortably for approximately 15 minutes with a dry cotton pledget placed inside one nostril (unilaterally) at the site of the deficient inferior turbinate without any anesthesia. Any changes in sensation or symptoms are recorded, following which the test is repeated on the other side.

3. Subsequently, the dry cotton pledget is substituted with another cotton pledget soaked in an epinephrine/xylocaine mixture, and the same tests are repeated. Any changes in nasal sensation or symptoms are recorded.

Comparison of the results of the aforementioned cotton tests can facilitate differentiation of a true ENS case from other disease entities such as allergic or atrophic rhinitis. This is because the nasal air fluency sensation would not generally improve in other conditions with the dry cotton; however, may significantly well respond to the epinephrine/xylocaine solution. In addition, testing each nose permits diagnosis of unilateral or bilateral ENS. As seen in our case, the presentation of ENS is not necessarily bilateral; however, the lack of nasal turbinate on one side can cause significant patient discomfort.

Surgical outcomes appear to be affected by the implantation site. A study reported that lateral nasal wall implantation might provide significantly better clinical outcomes than inferior nasal wall implantation [6]. Reconstruction of the missing inferior turbinates on the lateral wall creates a physiological pattern of airflow through the nasal passage closely resembling the natural structures.

Various materials have been used for nasal mucosal tissue expansion, including autologous materials (e.g., bone, cartilage, muscle, and fat), allografts materials (e.g., Teflon, Gore-tex, and Alloderm), and injectable materials (e.g., hyaluronic acid) $[1,2,7]$. Although, allografts demonstrate appreciable biocompatibility with surrounding tissues, the incidence of infection, extrusion, and rejection is higher than that of autologous materials. In our opinion, autologous materials are immune to rejection, extrusion, and infection should be the choice for implants.

A study evaluated 31 patients with ENS who underwent endonasal microplasty with conchal cartilage $(n=17)$ or costal cartilage implants $(n=14)$. The study concluded that while both materials demonstrated statistically significant improvements in the SNOT-25 score, the difference between pre- and post operative scores were higher in costal cartilage implants [6]. Costal cartilage has the advantage of providing sufficient tissue volume and durability [7].

Conchal cartilage is easy to handle, mold, and shape into the specific reconstruction design; however, may have to be harvested from both sides in cases of bilateral ENS. Although costal cartilage has been reported to provide optimal tissue volume for reconstruction, we believe that conchal cartilage also provides adequate tissue volume in cases of unilateral ENS, considering its ease of handling and moldability the resulting would and risk of complications from harvesting the graft is smaller. Autologous conchal cartilage offers a high level of biocompatibility and endurance with minimal risk of implant infection, translocation, extrusion, and rejection.

\section{Conclusion}

ENS is a rare disorder characterized by paradoxical nasal obstruction despite a broad nasal cavity, usually due to over-resection of the inferior turbinates. Surgical treatment for ENS aims to reconstruct the internal nasal structures. Nasal resistance is increased by narrowing the nasal cavity by reconstructing or 
augmenting the inferior turbinate. The choice of implantation site affects the outcomes. In our case, where the symptoms were limited to one side of the nose, we utilized autologous conchal cartilage for inferior turbinate augmentation and the patient achieved relief from the symptoms.

\section{Conflicts of interest}

The authors have nothing to disclose.

\section{References}

1. Houser SM. Surgical treatment for empty nose syndrome. Arch Otolaryngol Head Neck Surg 2007;133:858-63.

2. Jiang C, Shi R, Sun Y. Study of inferior turbinate reconstruction with Medpor for the treatment of empty nose syndrome. Laryngoscope 2013;123:1106-11.

3. Chen XB, Leong SC, Lee HP, Chong VF, Wang DY. Aerody- namic effects of inferior turbinate surgery on nasal airflow--a computational fluid dynamics model. Rhinology 2010;48:394400.

4. Sozansky J, Houser SM. Pathophysiology of empty nose syndrome. Laryngoscope 2015;125:70-4.

5. Thamboo A, Velasquez N, Habib AR, Zarabanda D, Paknezhad H, Nayak JV. Defining surgical criteria for empty nose syndrome: validation of the office-based cotton test and clinical interpretability of the validated Empty Nose Syndrome 6-Item Questionnaire. Laryngoscope 2017;127:1746-52.

6. Lee TJ, Fu CH, Wu CL, Lee YC, Huang CC, Chang PH, et al. Surgical outcome for empty nose syndrome: impact of implantation site. Laryngoscope 2018;128:554-9.

7. Jung JH, Baguindali MA, Park JT, Jang YJ. Costal cartilage is a superior implant material than conchal cartilage in the treatment of empty nose syndrome. Otolaryngol Head Neck Surg 2013;149:500-5. 